\title{
Reflective Optical Phase Modulator Based on High-Contrast Grating Mirrors
}

\author{
Yu Horie, Amir Arbabi, and Andrei Faraon \\ T. J. Watson Laboratory of Applied Physics, California Institute of Technology, 1200 E California Blvd, Pasadena, \\ CA 91125, USA \\ yhorie@caltech.edu
}

\begin{abstract}
We propose a reflective phase-only modulator formed by two layers of highcontrast grating reflectors. By arranging such optical phase modulators in a 2D array, ultra-fast Si-based phase-only spatial light modulators can be realized.
\end{abstract}

(C) 2014 Optical Society of America

OCIS codes: (050.6624) Subwavelength structures, (050.2770) Gratings, (230.6120) Spatial light modulators.

Deterministic spatio-temporal control of the phase of light propagating in free space is of great importance for many applications such as Fourier optics, holography or adaptive optics. Several phase modulation schemes have been incorporated into spatial light modulators (SLMs) using liquid crystal index modulation or movable mirrors based on MEMS actuation [1]. However, these phase modulation techniques operate slowly typically with a few $\mathrm{kHz}$ modulation bandwidth and involve relatively complicated fabrication processes. The spatial phase modulator analyzed here is based on traditional Gires-Tournois interferometers (GTI), in which the most of the incident light is reflected back with a strong phase delay dependent on the detuning from the GTI resonance.

The proposed reflective phase modulator, shown in Fig. 1(a), consists of two high contrast-grating (HCG) layers which are highly reflecive [2,3]. The HCG reflectors are designed to possess a nearly $100 \%$ reflectivity for TM polarized light at $1.55 \mu \mathrm{m}$. The top one has a slightly lower reflectivity than the bottom one. Owing to the thin thickness of the HCG layers, the GTI resonance can be efficiently tuned with respect to the wavelength of the incident light [4] by means of refractive index tuning of the HCG layers via the electro-optical (EO) or the thermo-optical (TO) effect.

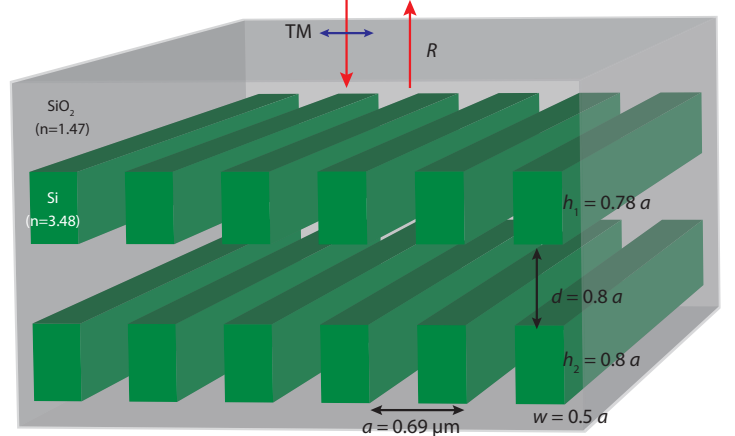

(a)

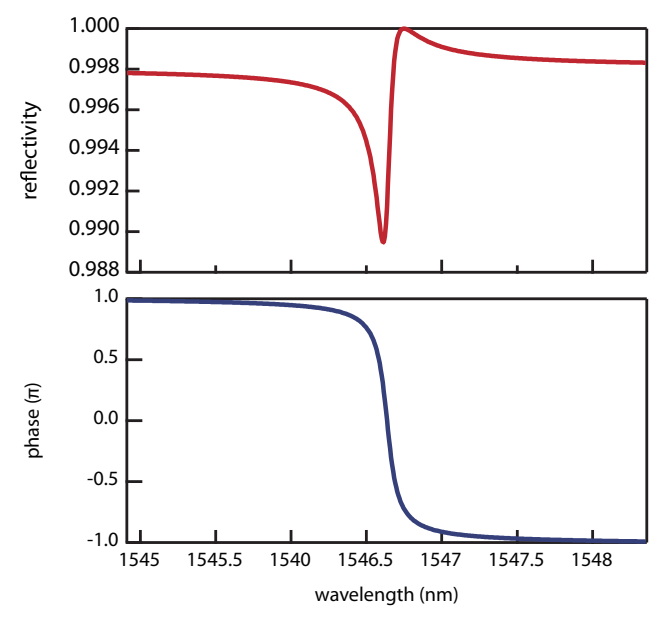

(b)

Fig. 1. (a) Schematic view of the proposed reflective phase modulator for TM polarization (electric field is perpendicular to the grating bar direction). The design is chosen such that only the thicknesses of the two layers are different, so the structure can be fabricated in one etch step. (b) The RCWAcalculated reflectivity spectrum, as well as the reflection phase as a function of wavelength. The resonator formed by an asymmetric two HCG reflectors has a quality factor of $10^{4}$ in the telecom band. Thanks to the asymmetric configuration of the two reflectors, the phase is very wavelength sensitive while the transmission loss is negligible. 


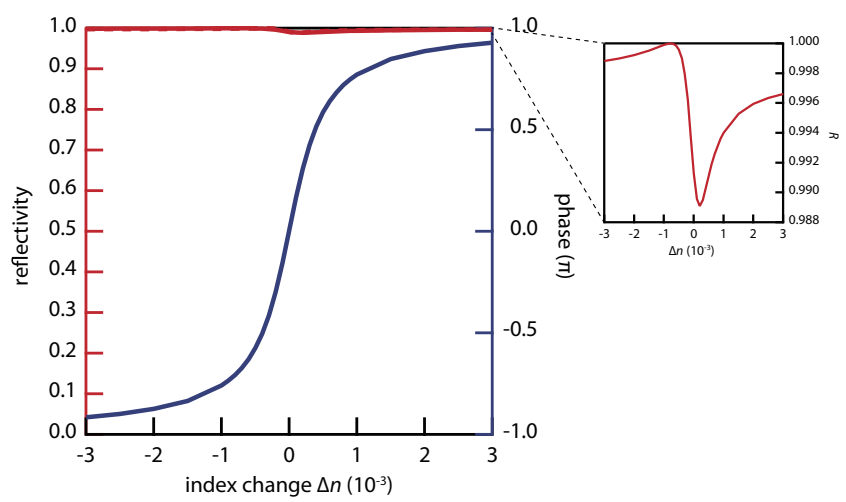

(a)

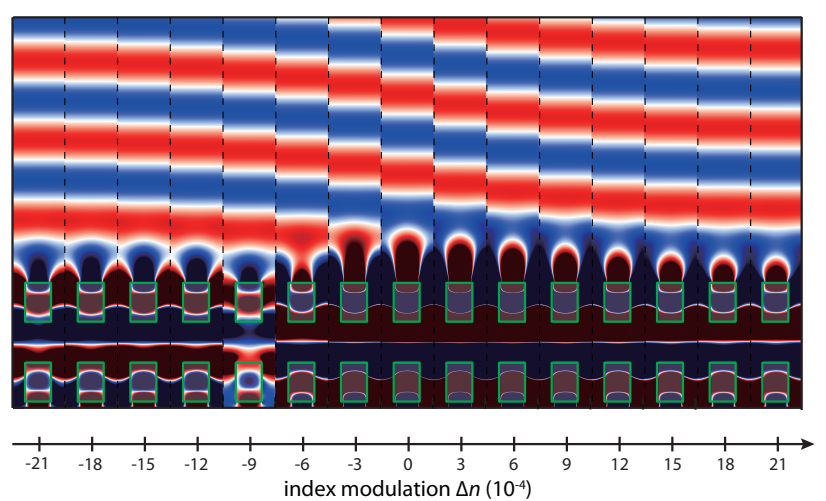

(b)

Fig. 2. Simulated operation of the designed reflective phase modulator. (a) The RCWA-calculated reflectivity for the wavelength $\lambda_{0}=1546.7 \mathrm{~nm}$ under the refractive index modulation only for the top Si HCG. (b) Series of snapshots for the reflected electric field emitted from the resonator (calculated via FDTD). The green circled areas are $\mathrm{Si}$, otherwise silicon dioxide.

Therefore, the choice of HCGs here is suitable for efficient and high-speed phase modulation. The reflectivity spectrum as well as the reflection phase for the double HCG reflectors used in the phase modulator was calculated using the rigorous coupled-wave analysis (RCWA) method [5], and are plotted in Fig. 1(b). The top and bottom reflectors with reflectivities of $R_{\text {top }}=0.998$ and $R_{\text {botom }}=0.999$, respectively, form a Fabry-Pérot (FP) resonance with a quality factor of $10^{4}$ around $1.55 \mu \mathrm{m}$. Because of the asymmetric mirror configuration, $99 \%$ of the incident light is reflected back, while the $\sim 2 \pi$ phase dispersion is built up.

We investigated the feasibility of modulating the phase of the reflected light by means of actively detuning the resonance. For the original resonance wavelength $\lambda_{0}=1546.7 \mathrm{~nm}$, the reflectivity as well as the imposed phase shift are calculated while changing the refractive index of only the top Si grating, as shown in Fig. 2(a). Nearly $2 \pi$-phase shift was obtained by detuning the resonance wavelength of the FP resonator with refractive index modulation of $\Delta n \sim 10^{-3}$. This refractive index modulation range we used is practically achievable via EO or TO effects in Si or other semiconductor materials. In Fig. 2(b), we show the phase front control of the reflected light emitted from the detuned resonator as obtained from the finite-difference time-domain (FDTD) simulations.

In conclusion, we proposed a reflective optical phase-only modulator based on a GTI configuration taking advantage of thin HCG reflectors. The phase modulation can be reasonably realized via index modulation of high index materials, thus allowing to realize high-speed modulation with very high bandwidth in the $\mathrm{MHz}$ or even $\mathrm{GHz}$ range. By placing such a phase modulator in a 2D array, phase-only SLMs working at a very high speed can be fabricated at low cost using the conventional CMOS processes.

\section{References}

1. W. Yang, T. Sun, Y. Rao, M. Megens, T. Chan, B.-W. Yoo, D. A. Horsley, M. C. Wu, and C. J. Chang-Hasnain, "High-speed optical phased array using high-contrast grating all-pass filters," in "International Conference on Indium Phosphide and Related Materials IPRM," (IEEE, 2012), pp. 22-24.

2. C. J. Chang-Hasnain and W. Yang, "High-contrast gratings for integrated optoelectronics," Adv. Opt. Photonics 4, 379-440 (2012).

3. C. Sciancalepore, B. B. Bakir, X. Letartre, J. Harduin, N. Olivier, C. Seassal, J. Fedeli, and P. Viktorovitch, "CMOS-compatible ultra-compact $1.55-\mu \mathrm{m}$ emitting VCSELs using double photonic crystal mirrors," IEEE Photonics Technol. Lett. 24, 455-457 (2012).

4. C. Qiu, J. Chen, Y. Xia, and Q. Xu, "Active dielectric antenna on chip for spatial light modulation," Sci. Rep. 2, 855 (2012).

5. V. Liu and S. Fan, "S4: A free electromagnetic solver for layered periodic structures," Comput. Phys. Commun. 183, 2233-2244 (2012). 